\title{
Treatment and prognosis of Masaoka stage 3 thymic carcinoma: a retrospective study of 32 cases
}

This article was published in the following Dove Press journal:

OncoTargets and Therapy

6 April 2015

Number of times this article has been viewed

Yan Sun ${ }^{1,2}$

Jinshi Liu ${ }^{1,3}$

Xinmin $\mathrm{Yu}^{1,2}$

'Key Laboratory Diagnosis and Treatment Technology on Thoracic Oncology, Hangzhou, People's Republic of China; ${ }^{2}$ Department of Medical Oncology, Zhejiang Cancer Hospital, Hangzhou, People's Republic of China; ${ }^{3}$ Department of Oncological Surgery, Zhejiang Cancer Hospital, Hangzhou, People's Republic of China

Correspondence: Yan Sun

Department of Medical Oncology, Zhejiang Cancer Hospital, 38 Guangji Road, Gongshu District, Hangzhou, 310022, People's Republic of China

Tel +8657188122182

Fax +86 571 8812 2188

Email zjch1971@।63.com
Purpose: The aim of this study was to investigate the treatment and prognostic factors in patients with Masaoka stage 3 thymic carcinoma.

Methods: A retrospective review was conducted of the medical records of patients with Masaoka stage 3 thymic carcinoma between 2000 and 2012 in our institution. Clinical characteristics and prognostic factors were analyzed. Survival curves were plotted using the Kaplan-Meier method. The Cox proportional hazard model was used for multivariate analysis.

Results: Thirty-two patients with Masaoka stage 3 thymic carcinoma, operated on in Zhejiang Cancer Hospital, were identified between 2000 and 2012. Among 32 patients, 24 achieved R0 resection. The most common histological subtypes were squamous cell carcinoma $(n=15,46.8 \%)$, followed by undifferentiated carcinoma $(n=12,37.5 \%)$, and other tumors $(\mathrm{n}=5,15.7 \%)$. The 5 -year disease-free survival and overall survival rates were $56.8 \%$ and $61.5 \%$, respectively. Patients with incomplete resection had a significantly worse disease-free survival and overall survival as compared to complete resection with univariate analyses ( $P$-value 0.006 and 0.034 , respectively). Multivariate analysis revealed that complete resection was statistically associated with disease-free survival but not overall survival ( $P$-value 0.025 and 0.076 , respectively).

Conclusion: Our results indicated that complete resection could impact the disease-free survival of patients with stage 3 thymic carcinoma.

Keywords: thymic carcinoma, treatment, prognosis, Masaoka stage 3

\section{Introduction}

Thymic carcinoma is a rare, aggressive neoplasm of the anterior mediastinum that is morphologically and biologically distinct from thymoma. ${ }^{1}$ Surgery was the main therapeutic modality for the early stage thymic carcinoma patients. ${ }^{2}$ Because of the involvement of surrounding vital structures, the treatment and prognosis of Masaoka stage 3 thymic carcinoma was different from stage 1 and 2 patients.

In previous studies, both thymoma and thymic carcinoma were included and analyzed, reports focusing on thymic carcinoma were limited. ${ }^{3-6}$ The value of resection, radiotherapy, and chemotherapy has remained uncertain for the Masaoka stage 3 thymic carcinoma until now. ${ }^{7-9}$ The optimal treatment for Masaoka stage 3 thymic carcinoma needs to be defined.

In the present study, we retrospectively evaluated the treatment and prognosis in patients with Masaoka 3 thymic carcinoma, and explored the role of different prognosis factors in this setting. 


\section{Patients and methods Methods}

A total of 82 patients had surgical resection for thymic carcinoma between January 2000 and January 2012 in Zhejiang Cancer Hospital. The Ethics Committee at Zhejiang Cancer Hospital approved the study. The stage of thymic carcinoma was classified based on the Masaoka staging system. Thirtytwo patients were diagnosed as pathologically Masaoka stage 3 in this period. All of the 32 patients with resection had pathologically confirmed thymic carcinoma. Histologic subtypes were determined by 2004 World Health Organization (WHO) histologic classification and two pathologists (Professor Wenyong Sun and Haimiao Xu) reviewed and confirmed diagnosis of all the thymic carcinomas. The recurrence or metastases were confirmed using chest computerized tomography (CT), as well as ultrasound and/or CT of the abdomen.

\section{Follow-up}

Surviving patients were followed-up every 3 to 6 months for the first 5 years, then annually. The last censoring date for survival was September 2013. The median follow-up of patients was 68 months ranging from 20 to 189 months.

\section{Statistical analysis}

The statistical analysis using the SPSS version 16 (SPSS Inc., Chicago, IL, USA) was performed, assuming that $P$-value less than 0.05 is statistically significant. The survival curves were generated using the Kaplan-Meier method. Disease-free (DFS) survival encompassed the time from the surgery to documented progression or death from any cause. The definition of overall survival (OS) was determined from the date of surgery and the last known follow-up or date of death.

\section{Results}

\section{Patient characteristics}

Table 1 shows the patient clinical and histologic characteristics. The median age was 47 years in all of the patients (range, 22-71 years). Fifteen patients were diagnosed with squamous cell carcinoma and 12 patients with undifferentiated carcinoma. Four patients were diagnosed with neuroendocrine tumors and one patient with mucoepidermoid carcinoma.

\section{Treatment}

All patients underwent surgical resection. Twenty-four patients received complete resection and eight received incomplete resection. Twenty-one patients were treated
Table I Demographic characteristics of the study population

\begin{tabular}{ll}
\hline & Number (\%) \\
\hline Sex & \\
Male & $15(46.9)$ \\
Female & $17(53.1)$ \\
Age & \\
Range & $22-7 \mid$ \\
Median & 47 \\
$<50$ & $17(53.1)$ \\
$\geq 50$ & $15(46.9)$ \\
Histology & \\
Squamous cell carcinoma & $15(46.9)$ \\
Undifferentiated carcinoma & $12(37.5)$ \\
Neuroendocrine tumor & $4(12.5)$ \\
Others & $1(3.1)$ \\
Resection & \\
Complete & $24(75)$ \\
Incomplete & $8(25)$ \\
Myasthenia gravis & \\
Yes & $4(12.5)$ \\
No & $28(87.5)$ \\
Treatment after surgery & \\
Only radiotherapy & $7(21.9)$ \\
Only chemotherapy & $5(15.6)$ \\
Radiotherapy + chemotherapy & $14(43.8)$ \\
No & $6(18.7)$ \\
\hline
\end{tabular}

with radiation therapy (prescribed dose ranged from 40 Gy to $60 \mathrm{~Gy}$ ). Nineteen patients received chemotherapy (five received only chemotherapy, 14 received radiotherapy and chemotherapy). Of the patients with incomplete resection, four had recurrence of local lesions and two had metastasization (one with lung and one with bone). Second operations were performed after recurrence of local lesions in three patients.

\section{Survival and prognostic factors}

The mean time of postoperative recurrence occurred at 101 months after initial surgery. The DFS rate was $56.8 \%$ at 5 years and $48.7 \%$ at 10 years. The OS rate at 5 years was $61.5 \%$, and at 10 years it was $36.9 \%$.

Univariate analyses were performed by the KaplanMeier method to assess the predictive capability of each variable assessed. These data are summarized in Table 2 . Age, sex, radiotherapy, chemotherapy, and myasthenia gravis were not found to be statistically associated with DFS and OS. Complete resection was predictive of DFS and OS (Figures 1 and 2).

The variables included in the final model are presented in Table 3 and include age, sex, radiotherapy, chemotherapy, complete resection, myasthenia gravis, and histology, complete resection has significantly influenced DFS but not OS in multivariate analysis. 
Table 2 Univariate analysis of DFS and OS in 32 thymic carcinoma patients

\begin{tabular}{|c|c|c|c|c|}
\hline & 5-year DFS rate (\%) & $P$ & 5-year OS rate (\%) & $P$ \\
\hline Sex & & 0.719 & & 0.656 \\
\hline Male & 51.4 & & 54.5 & \\
\hline Female & 61.2 & & 66.6 & \\
\hline Age & & 0.317 & & 0.079 \\
\hline$<50$ & 52.8 & & 60.0 & \\
\hline$\geq 50$ & 61.8 & & 80.2 & \\
\hline Histology & & 0.752 & & 0.318 \\
\hline Squamous cell carcinoma & 49.7 & & 60.0 & \\
\hline Others & 61.9 & & 69.5 & \\
\hline Complete resection & & 0.006 & & 0.034 \\
\hline Yes & 65.3 & & 70.8 & \\
\hline No & 30.0 & & 38.1 & \\
\hline Adjuvant chemotherapy & & 0.646 & & 0.256 \\
\hline Yes & 54.0 & & 54.5 & \\
\hline No & 59.8 & & 69.2 & \\
\hline Adjuvant radiotherapy & & 0.878 & & 0.907 \\
\hline Yes & 60.6 & & 63.7 & \\
\hline No & 55.1 & & 58.4 & \\
\hline Myasthenia gravis & & 0.548 & & 0.662 \\
\hline Yes & 50.0 & & 75.0 & \\
\hline No & 57.1 & & 60.1 & \\
\hline
\end{tabular}

Abbreviations: DFS, disease-free survival; OS, overall survival.

\section{Discussion}

Considering the rarity of the disease, this report of 32 cases is to be regarded as a relatively large study. Our results suggest that complete resection could impact the DFS but not OS with Masaoka stage 3 thymic carcinoma.

There are conflicting views in the literature with regard to the optimal treatment of thymic carcinoma. ${ }^{6-10}$ The efficacy of resection for thymic carcinoma has not yet been well studied until now. Some studies have indicated that complete resection was the most important factor related to the prognosis of patients with thymic carcinoma. ${ }^{11}$ Zhao et al showed that complete resection not only predicts DFS

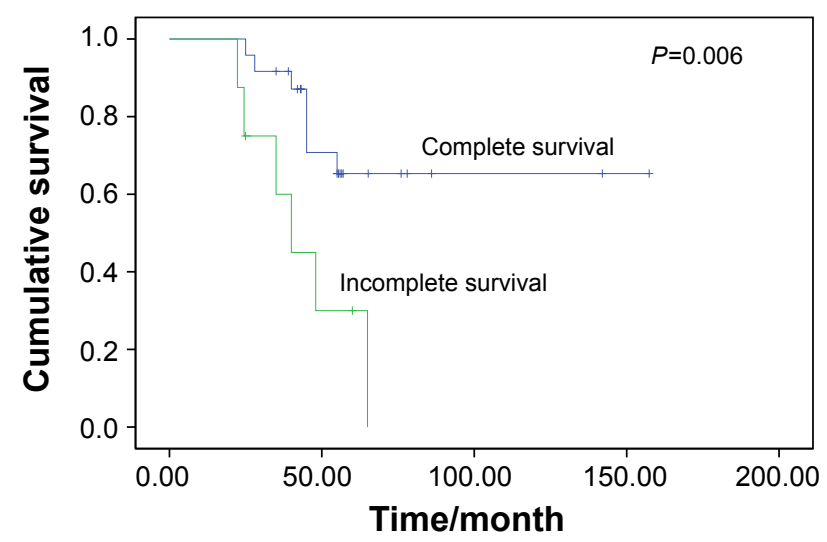

Figure I Comparison of disease-free survival between complete resection and incomplete resection patients $(P=0.006)$. but also OS after resection of 105 thymic squamous cell carcinomas. ${ }^{12}$ However, some reports have showed that there was no survival difference for patients who underwent complete resection compared with incomplete resection. ${ }^{9,13}$ In the current study, there was DFS and OS differences between complete and incomplete resection patients with univariate analyses. However, the difference of OS was not significant by multivariate analysis, the reason may be due to the small number of patients in our study.

It remains controversial whether adjuvant radiation and chemotherapy reduces the rate of local recurrence and distant metastasis. Fan et al showed potential advantages in improving

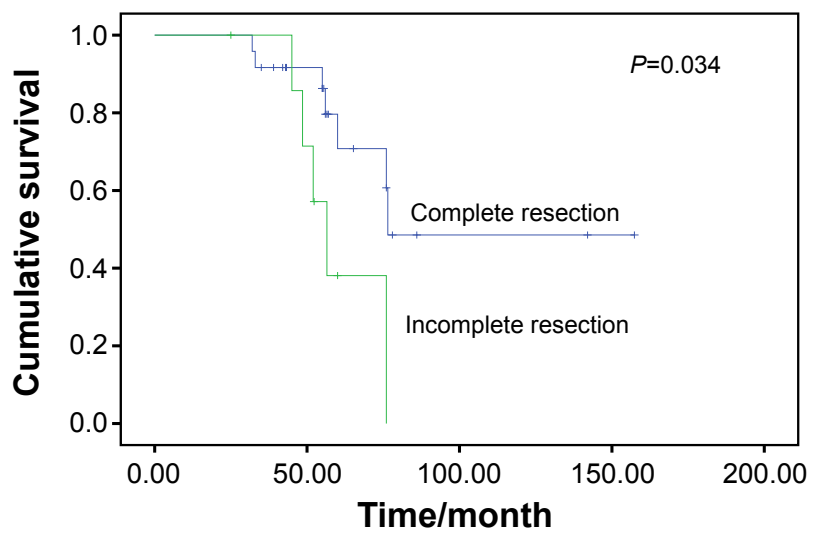

Figure 2 Comparison of overall survival between complete resection and incomplete resection patients $(P=0.034)$. 
Table 3 Multivariate survival analysis for disease-free survival (DFS) and overall survival (OS)

\begin{tabular}{|c|c|c|c|c|c|c|}
\hline & \multicolumn{3}{|l|}{ DFS } & \multicolumn{3}{|l|}{ OS } \\
\hline & HR & $95 \% \mathrm{Cl}$ & $P$ & HR & $95 \% \mathrm{Cl}$ & $\boldsymbol{P}$ \\
\hline Age $(\geq 50$ vs $<50)$ & 1.421 & $0.211-4.512$ & 0.514 & 2.431 & $0.311-10.4211$ & 0.421 \\
\hline Complete resection (yes vs no) & 0.215 & $0.015-0.879$ & 0.025 & 0.452 & $0.142-1.075$ & 0.076 \\
\hline Adjuvant chemotherapy (yes vs no) & 2.741 & $0.512-16.542$ & 0.425 & 2.042 & $0.76|-| 4.214$ & 0.765 \\
\hline Histology (squamous vs others) & 0.578 & $0.165-2.879$ & 0.425 & 0.341 & $0.078-1.987$ & 0.541 \\
\hline
\end{tabular}

Abbreviations: $\mathrm{HR}$, hazard ratio; $\mathrm{Cl}$, confidence interval; vs, versus.

survival and reducing relapse in patients with Masaoka stage 3 thymoma with radiotherapy. ${ }^{14}$ However, a study by the Japanese Association for Chest Surgery demonstrated that neither adjuvant radiation nor chemotherapy improved OS in patients with completely resected thymic carcinoma. ${ }^{15}$ Our series also revealed that adjuvant treatment did not have a significant effect on Masaoka stage 3 thymic carcinoma. However, considering selection bias and small number of patients, no conclusion can be set on the efficacy of adjuvant treatment.

Our study is limited by its retrospective design over a long period and its small number of patients. In addition, there were only eight patients with incomplete resection. Therefore, the influence of resection on DFS and OS must be considered carefully. Thirdly, neoadjuvant treatment showed a high efficacy for some locally advanced thymic carcinomas and increased complete resection rate, while, no neoadjuvant cases were enrolled in the current study. However, with few cases in previous clinical studies, our retrospective study may also be considered to be meaningful.

In conclusion, complete resection provided remarkable DFS benefit in Masaoka stage 3 thymic carcinoma. The role of radiotherapy and chemotherapy need to validate in the prospectively large studies.

\section{Disclosure}

The authors have no conflicts of interest to disclose.

\section{References}

1. Engels EA. Epidemiology of thymoma and associated malignancies. J Thorac Oncol. 2010;5(10 Suppl 4):S260-S265.
2. Margaritora S, Cesario A, Cusumano G, et al. Thirty-five-year follow-up analysis of clinical and pathologic outcome of thymoma surgery. Ann Thorac Surg. 2010;89(1):245-252.

3. Venuta F, Anile M, Diso D, et al. Thymoma and thymic carcinoma. Eur J Cardiothorac Surg. 2010;37(1):13-25.

4. Demirci S, Turhan K, Ozsan N, et al. Prognostic factors for survival in patients with thymic epithelial tumors. Thorac Cardiovasc Surg. 2011;59(3):153-157.

5. Suster S, Rosai J. Thymic carcinoma: a clinicopathologic study of 60 cases. Cancer. 1991;67(4):1025-1032.

6. Lucchi M, Mussi A, Ambrogi M, et al. Thymic carcinoma: a report of 13 cases. Eur J Surg Oncol. 2001;27(7):636-640.

7. Forquer JA, Rong N, Fakiris AJ, Loehrer PJ Sr, Johnstone PA. Postoperative radiotherapy after surgical resection of thymoma: Differing roles in localized and regional disease. Int J Radiat Oncol Biol Phys. 2010;76(2):440-445.

8. Weide LG, Ulbright TM, Loehrer PJ Sr, Williams SD. Thymic carcinoma: a distinct clinical entity responsive to chemotherapy. Cancer. 1993; 71(4):1219-1223.

9. Shen S, Ai X, Lu S. Long-term survival in thymic epithelial tumors: a single-center experience from China. J Surg Oncol. 2013; 107(2):167-172.

10. Song Z, Zhang Y. Adjuvant therapy in stage II thymic carcinoma. J Cancer Res Clin Oncol. 2014;140(2):349-352.

11. Yano M, Sasaki H, Yokoyama T, et al. Thymic carcinoma: 30 cases at a single institution. J Thorac Oncol. 2008;3(3):265-269.

12. Zhao Y, Zhao H, Hu D, et al. Surgical treatment and prognosis of thymic squamous cell carcinoma: a retrospective analysis of 105 cases. Ann Thorac Surg. 2013;96(3):1019-1024.

13. Blumberg D, Burt ME, Bains MS, et al. Thymic carcinoma: current staging does not predict prognosis. J Thorac Cardiovasc Surg. 1998; 115(2):303-309.

14. Fan C, Feng Q, Chen Y, et al. Postoperative radiotherapy for completely resected Masaoka stage III thymoma: a retrospective study of 65 cases from a single institution. Radiat Oncol. 2013;8:199.

15. Kondo K, Monden Y. Therapy for thymic epithelial tumors: a clinical study of 1,320 patients from Japan. Ann Thorac Surg. 2003;76(3): 878-884.
OncoTargets and Therapy

\section{Publish your work in this journal}

OncoTargets and Therapy is an international, peer-reviewed, open access journal focusing on the pathological basis of all cancers, potential targets for therapy and treatment protocols employed to improve the management of cancer patients. The journal also focuses on the impact of management programs and new therapeutic agents and protocols on

\section{Dovepress}

patient perspectives such as quality of life, adherence and satisfaction. The manuscript management system is completely online and includes a very quick and fair peer-review system, which is all easy to use. Visit http://www.dovepress.com/testimonials.php to read real quotes from published authors. 